\title{
Análisis de la gestión del talento en función de sus dimensiones y procesos. Un estudio de casos*
}

\author{
Analysis of talent management according to its dimensions and processes. A case study \\ Análise da gestão do talento em função de suas dimensões e processos. Um estudo de casos
}

Maria del Carmen de la Calle-Durán ${ }^{\text {a }}$

Universidad Rey Juan Carlos, España

DOI: https://doi.org/10.11144/Javeriana.cao33.agtfdp

carmen.delacalle@urjc.es

ORCID: http://orcid.org/0000-0003-3368-8596

Recepción: 25/04/2019

Fernando Enrique García Muiña

Aceptación: 03/12/2019

Universidad Rey Juan Carlos, España

ORCID: http://orcid.org/0000-0002-7807-3715

Alvaro Alonso Osuna

Universidad Rey Juan Carlos, España

ORCID: http://orcid.org/0000-0002-7797-8045

\section{Resumen:}

La gestión del talento es, hasta la fecha, un campo de estudio ambiguo donde coexisten cuatro vías de enfoque diferenciadas: la primera la considera como un proceso similar a la gestión de personal cualificado; la segunda, básicamente, relaciona la gestión del talento con la planificación y sucesión de empleados; la tercera, defiende el desarrollo del personal de mayor rendimiento; y la cuarta, vincula dicho desarrollo con los puestos clave. El objetivo de este trabajo es analizar las dimensiones y los procesos de la gestión del talento, y estudiar su relación con dichas vías de enfoque. Para ello, fue realizado un estudio de casos de cuatro empresas que operan en España, lo que nos ha permitido concluir que las vías de enfoque no son excluyentes.

Código JEL: M12

Palabras clave: Gestión del talento (GT), talento humano, dimensiones y procesos de la GT.

\section{Abstract:}

Talent management is, to date, an ambiguous field of study where four different approaches coexist: the first considers it similar to the management of qualified personnel; the second relates talent management with planning and succession of employees; the third defends the development of higher-performing personnel; and the fourth links this development with key positions. This work aims to analyze the dimensions and processes of talent management and to study their relationship with these lines of approach. To this end, a case study of four companies operating in Spain has been carried out, which has allowed us to conclude that the approaches are not exclusive.

JEL Codes: M12

Keywords: Talent Management (TM), human talent, TM dimensions and processes.

\section{Resumo:}

A gestão do talento é, até hoje, um campo de estudo ambíguo aonde coexistem quatro vias de enfoque diferenciadas: a primeira a considera como um processo similar à gestão do pessoal qualificado; a segunda, basicamente, relaciona a gestão do talento com a planificação e sucessão de empregados; a terceira, defende o desenvolvimento do pessoal de maior rendimento; a quarta, vincula tal desenvolvimento como os postos chaves. O objetivo deste trabalho é analisar as dimensões e os processos da gestão do talento, e estudar sua relação com tais vias de enfoque. Para isso, foi realizado um estudo de casos de quatro empresas que operam em Espanha, o que nos tem permitido concluir que as vias de enfoque não são excludentes.

Código JEL: M12

Palavras-chave: Gestão do talento (GT), talento humano, dimensões e processos da GT.

Notas de autor

\footnotetext{
${ }^{\text {a }}$ Autora de correspondencia. Correo electrónico: carmen.delacalle@urjc.es
} 


\section{Introducción}

La terminología empleada en el campo de investigación de la Gestión del Talento -GT- es extensa, confusa y ambigua. Debido a su habitual inclusión dentro del ámbito de los recursos humanos, tanto su objeto de estudio como las denominaciones de las variables involucradas en los modelos de análisis a lo largo del tiempo han sido diversos, y han evolucionado con la propia disciplina de los recursos humanos. Por ello, se ha de analizar en detalle el objeto de estudio de las investigaciones para confirmar su pertenencia al ámbito de la GT (Dries, 2013), ya que es común encontrar trabajos en los que se hace referencia a este proceso para todo aquello relacionado con el Capital Humano, sin que sea específico de la GT. Tal es el caso, por ejemplo, de las investigaciones orientadas a las relaciones con los sindicatos (Meyers, van Woerkom y Dries, 2013). De igual manera, mientras que en ocasiones la GT se considera como una disciplina independiente, en otras es considerada tan solo como una habilidad directiva más (Lewis y Heckman, 2006). Por todo ello, Dries (2013) defiende que ese mismo hecho dota al campo de una flexibilidad inusitada, sobre la que se asienta su elevada diversidad, así como las múltiples y diferentes aplicaciones del término.

Una de las principales fuentes de divergencia conceptual es la apreciación que de la palabra talento se hace en los países anglosajones frente a los mediterráneos. Lógicamente, este matiz que posee la palabra talento afecta también al término GT, profundizando en la confusión dependiendo del área geográfica desde la que se trate el asunto. Por ello, la mayoría de trabajos que, desde un enfoque latino-mediterráneo, se aproxima a la GT, lo hace teniendo en consideración el nivel educativo alcanzado, mientras que, en los estudios procedentes de países anglosajones, el término se relaciona con aquellos individuos que son capaces de marcar diferencias y crear ventajas competitivas sostenibles, independientemente de su nivel educativo o su cualificación profesional (Dries, 2013).

Lewis y Heckman (2006) hacen un profundo estudio de las diferentes aproximaciones teóricas a la GT, en el que concluyen la existencia de tres corrientes o vías principales de aproximación al campo. A éstas debe adicionarse una cuarta vía desarrollada posteriormente por Collings y Mellahi (2009). La primera vía utiliza el término como si se tratara de un sinónimo de gestión de personal cualificado (enfoque mediterráneolatino). Según esta vía, las estrategias y tácticas a emplear para la GT son las mismas que se utilizan para los recursos humanos de manera genérica. De esta manera, la GT facilitará y agilizará la gestión del personal cualificado de la organización. Por su parte, la segunda vía define la GT desde un punto de vista estrictamente cuantitativo o estadístico. Para ello, presta atención al estudio de las entradas y salidas del personal cualificado sin entrar a valorar el alto o bajo rendimiento del personal considerado como "talento". Tan solo se enfoca en las probabilidades de que se mantenga o no en la empresa, así como en los medios empleados para agilizar su reposición. La tercera vía considera que la GT pretende identificar y desarrollar, a través de un conjunto de prácticas, el personal de más alto rendimiento considerado como recurso estratégico y fuente de ventajas competitivas.

Collings y Mellahi (2009) aportarían una corriente adicional o cuarta vía, que centra su atención no tanto en los individuos de alto potencial de la empresa, sino en los puestos clave de la compañía. Es decir, la GT reside en el análisis de los puestos de trabajo sobre los que se asientan las ventajas competitivas y no tanto en las personas "talentosas" que puede haber a lo largo de la organización. Se considera que el factor humano solamente será fuente de ventajas competitivas cuando ocupe puestos de trabajo que resulten clave para la organización. En este sentido, esta vía establece diferencias sustanciales entre lo que sería la GT y la gestión de recursos humanos, extendida a todos los puestos de la organización y a todo su personal. Si, por un lado, la dirección de recursos humanos clásica no discrimina a la hora de hacer frente a los problemas relacionados con las personas y las posiciones dentro de la empresa, la GT dedica sus esfuerzos a diseñar actividades y procesos específicos adaptados a las personas que la empresa identifica como altos potenciales, así como a los puestos clave estratégicamente identificados (Alonso y García-Muiña, 2014). De esta forma se identifican dos dimensiones de la GT: las personas y los puestos claves. La consideración de una u otra dimensión lleva a un 
tratamiento diferencial sobre las actividades o procesos clásicos de gestión de los recursos humanos, y a los mecanismos de implementación.

En este sentido, los objetivos de este trabajo son, a partir del análisis de un estudio de casos: (i) analizar si coexisten en las empresas las cuatro vías teóricamente establecidas, y (ii) determinar la relación que existe entre las dimensiones de la GT y estas cuatro vías de estudio.

Las principales contribuciones del trabajo son, en primer lugar, el análisis bibliográfico de las diferentes vías de enfoque de la GT existentes en la literatura. En segundo lugar, se estudia la existencia de estas diferentes vías a través de la realidad empresarial, para evaluar la importancia que las empresas dan a las dimensiones y procesos de la GT. Todo ello es contrastado con un estudio empírico en un campo que, hasta la fecha, está dominado por los trabajos teóricos, especialmente en el caso español donde existen pocas investigaciones previas (Gallardo-Gallardo, Nijs, Dries, y Gallo, 2015).

Por ello, el trabajo tiene notables implicaciones organizativas y sociales. Para las empresas, la GT permite establecer las pautas de crecimiento y desarrollo de su talento humano, lo que favorecería su sostenibilidad organizativa en el largo plazo. Por su parte, la sociedad se ve favorecida porque aumenta el conocimiento y, por tanto, el capital humano ya que las empresas, a través de la GT, logran una mayor competitividad mediante procesos específicos de desarrollo de sus empleados de los que ellos también se ven favorecidos en su carrera profesional.

\section{Las cuatro vías de estudio en la GT}

Es posible explicar la diversidad de las vías de estudio de la GT por el hecho de que los diferentes autores la abordan desde su propio campo de investigación. Esto puede provocar un cierto sesgo a la hora de definirla y tratarla (Lewis y Heckman, 2006).

Salvo en el caso de la tercera vía, el resto contempla la GT desde un punto de vista unidimensional, limitando su papel a facilitar la implantación de la estrategia empresarial. Sin embargo, la tercera vía identifica a los individuos de alto potencial y rendimiento que pueden ocupar puestos clave, y adicionalmente reconoce que la GT no solo facilita el proceso de implantación estratégica, sino que orienta la formulación de nuevas estrategias empresariales al respecto. $\mathrm{Al}$ analizarlas separadamente, podemos darnos cuenta de cómo diferentes académicos aproximan la GT a su campo de trabajo habitual. Esto explica por qué cada una de estas vías estudia la GT de una forma diferente, aportando aplicaciones prácticas separadas unas de otras.

\section{Primera vía de estudio en la GT}

La primera vía identifica a la GT con la gestión de personal cualificado, por lo que las tácticas a emplear en este caso serán las mismas que clásicamente se han considerado desde la dirección de recursos humanos, pero para el personal de alta cualificación. Esta corriente defiende hacer lo mismo que la función de recursos humanos, pero mejor y más rápido cuando se trata de personal cualificado. Así, Lewis y Heckman, (2006, p. 141) se refieren al "talento como un eufemismo de personas". Además, este enfoque no estudia la relación existente entre la cualificación profesional y el desempeño organizativo de las personas que ocupan diferentes puestos de trabajo. Según esta vía, los puestos en la industria se catalogan mediante una serie de competencias y conocimientos. La función de la GT es asegurar el adecuado flujo de conocimientos y competencias a través de la organización.

Esta perspectiva es la que suelen utilizar los especialistas en recursos humanos cuando se adentran en la GT. Lewis y Heckman (2006) hacen referencia a este sesgo presente en los estudios, al mostrar cómo se trata de una postura defendida por autores que ya son especialistas en subdisciplinas de los recursos humanos. Así, especialistas en selección de personal centran la GT en cómo encontrar a los mejores candidatos posibles 
(Sullivan, 2005); especialistas en formación y desarrollo ponen especial hincapié en hacer crecer el talento (Cohn, Khurana, y Reeves, 2005); expertos en compensación y beneficios centran la GT en la gestión de las compensaciones por rendimiento (Garger, 1999); y expertos en liderazgo enfatizan el desarrollo de nuevos líderes y la organización de planes de sucesión (Conger y Fullmer, 2003).

\section{Segunda vía de estudio en la GT}

Como ya se adelantó, la segunda vía aplica el término GT a la gestión estadística de las entradas y salidas de personal cualificado o líderes, de acuerdo con las previsiones internas y externas de necesidades de personal. Por lo que la GT, para los autores que desarrollan sus trabajos en esta perspectiva, es un conjunto de procesos diseñados para asegurar un adecuado flujo de profesionales en la organización. Esta vía, al igual que la primera, centra su importancia estratégica en las personas, pero no lo hace desde el punto de vista de los procesos o tareas organizativas, sino desde un punto de vista estadístico.

Esta visión de la GT se acerca a lo que se conoce como planes de sucesión (Jackson y Schuler, 1990; Rothwell, 1994), aunque también incluye herramientas típicas de recursos humanos, como por ejemplo la selección de personal. Un desarrollo típico de un plan de talento de estas características implica la realización de un cálculo de necesidades de personal en los diferentes puestos, y el desarrollo de planes de carrera que permitan a los individuos ir avanzando por las líneas de sucesión de la empresa. En ocasiones, se utilizan sistemas basados en tecnologías de la información que guían las carreras de los empleados, según se van desarrollando sus perfiles y van quedando vacantes disponibles (Becker y Huselid, 2006).

Huselid y Becker (1999), Huselid, Beatty y Becker (2005) y Becker y Huselid (2006) ponen de manifiesto el enfoque industrial que poseen sus análisis de gestión estratégica de recursos humanos. Según estos autores, este sistema se ajusta a empresas dependientes de altas cantidades de personal cualificado entrando y saliendo todos los años, como pueden ser empresas tecnológicas e innovadoras que apuesten por la $\mathrm{I}+\mathrm{D}+\mathrm{i}$. En estas empresas, por ejemplo, asegurar el reemplazo de cientos de ingenieros cada año es una necesidad, por lo que planificar su constante flujo es la clave del éxito para que los programas de $\mathrm{I}+\mathrm{D}$ siempre cuenten con el personal necesario, ya que se entiende al personal cualificado como un recurso escaso. La utilización del personal como un recurso acerca posturas con la Teoría de Recursos y Capacidades (Barney, 1991; Grant, 1991) que considera al personal cualificado como un recurso con el que se construyen las capacidades de la empresa.

Hemos determinado que para esta segunda vía las personas son un recurso, si bien tiene la particularidad de que su control escapa muchas veces a la organización, de acuerdo con un flujo o corriente demográfica exógenamente definida (Scullion, Collings y Caligiuri, 2010). Por tanto, el contenido de la GT se limita a mitigar los efectos de un exceso o escasez de profesionales con las competencias requeridas.

\section{Tercera vía de estudio en la GT}

La tercera vía es la que reúne más literatura. Se centra en el análisis de necesidades de personal y de puestos clave, así como en la preparación del personal que más alto rendimiento demuestra, independientemente de la cualificación profesional que se posea. Los estudios se orientan a la cualificación de las capacidades de los sujetos para su mejor empleo dentro de la empresa, a fin de ayudar a crear valor (Walker y LaRocco, 2002).

Esta tercera vía entiende el talento de forma diferente a las dos anteriores. Según este enfoque, el talento no lo constituye todo el personal que forma parte de la empresa, ni siquiera el personal más cualificado o el personal en puestos directivos. El talento estaría compuesto por los individuos que muestran un alto potencial o rendimiento en el presente. Se trata de determinadas personas, que desempeñan roles diversos en cualquier 
parte de la organización y que implican un elevado valor añadido. Por tanto, no bastan personas con una cierta cualificación general para entrar en de la definición (Axelrod, Handfield-Jones y Michaels, 2002).

El fondo de esta corriente es el aporte de valor a los resultados de la compañía, a través del aprovechamiento de altos potenciales como generadores de nuevas estrategias. La idea es que este talento, desarrollado con autonomía y recursos, puede generar de forma espontánea ventajas competitivas. Esto gracias a que estas personas pueden aportar en la generación de nuevos procesos, nuevas tecnologías o técnicas de comercialización.

A su vez, dentro de esta vía encontramos dos propuestas diferentes en cuanto al desarrollo. Por un lado, una primera corriente de estudios defiende que estas personas, independientemente de su cualificación o de su puesto de trabajo, merezcan ser tratadas de forma diferente al resto de sus compañeros, existiendo para ellos planes diferenciados de supervisión, contratación y compensación (Axelrod et al., 2002). Los perfiles se identifican dentro de la empresa y se contratan del exterior, por lo que esta corriente no centra sus esfuerzos en el desarrollo del personal. De igual manera, y aquí es donde esta corriente aporta más valor a las organizaciones, ese trato diferenciado no debe depender de las necesidades para cubrir posiciones clave dentro de la compañía, sino del verdadero rendimiento de los profesionales.

La segunda corriente justifica que es labor de los departamentos de recursos humanos desarrollar a todos los empleados para que alcancen a ser altos potenciales o perfiles que demuestren alto rendimiento (Walker y LaRocco, 2002). Es decir, convertir al personal de la empresa no catalogado en trabajadores de alto rendimiento o potencial, para desarrollarse como los mejores, más que adquirir estos perfiles en el mercado laboral exterior. El fin último es permitir que la empresa se dote íntegramente de empleados de estas características, para que generen de manera espontánea nuevas ventajas competitivas.

En general, el objetivo final de ambas corrientes y su propuesta de valor es permitir mediante la elevación del potencial humano de la compañía, la aparición espontánea de nuevas capacidades que generen ventajas competitivas. Esto asegura a la compañía su perdurabilidad en el tiempo. Este enfoque de GT permite a las empresas no solo la implantación de estrategias, sino también su formulación.

\section{Cuarta vía de estudio en la GT}

A estas tres vías propuestas por Lewis y Heckman en 2006, hay que añadir una cuarta vía desarrollada por Collings y Mellahi en 2009 y Capelli (2008). Collings y Mellahi (2009) proponen una visión de la GT muy próxima a la de Lewis y Heckman (2006) en su tercera vía, pero en lugar de centrar su enfoque en los individuos de alto potencial o rendimiento, lo hacen en el análisis de las posiciones clave de la organización. Según estos autores, el objetivo de todo plan de GT en la empresa es asegurar el mantenimiento a largo plazo de las ventajas competitivas de primer orden de la compañía. El análisis, por tanto, debe hacerse en cascada partiendo de la identificación de las ventajas competitivas de cada empresa, para luego vincularlas con los puestos clave que las generan. A continuación, el plan debe contemplar la retención o el reemplazo adecuado en esas posiciones, contando para ello con los individuos de alto potencial de la empresa.

En realidad, esta cuarta vía podría definirse como una combinación de la tercera y la segunda. Por un lado, comparte con la segunda vía la disposición de planes de sucesión para evitar la ausencia de profesionales preparados en desempeñar funciones clave dentro de la empresa. Sin embargo, como sucede en la tercera vía, este proceso se realiza de forma selectiva, es decir, no considerando a todo el personal de la empresa sino sólo a las personas capaces de ocupar posiciones clave, y basándose en el potencial, no en las competencias. El hecho diferencial que hace novedosa esta perspectiva es que la GT no se hace alrededor de las personas sino alrededor de las ventajas competitivas de la empresa. 


\section{Análisis comparado de las vías de estudio y las dimensiones de la GT}

Para poder identificar los factores que afectan directamente a cada una de las vías, y evaluar si predomina alguna en nuestro posterior estudio de casos, elaboramos la tabla 1. En ésta se propone una descripción comparada de las diferentes vías de estudio de la GT en términos de las dimensiones y los procesos tratados.

TABLA 1

\begin{tabular}{|c|c|c|c|c|}
\hline Dimensión & Via I & Via II & Via III & Via IV \\
\hline Personas & $\begin{array}{l}\text { Considera la } \\
\text { mejora de las } \\
\text { técnicas clásicas } \\
\text { de recursos } \\
\text { humanos para el } \\
\text { personal de alta } \\
\text { cualificación }\end{array}$ & $\begin{array}{l}\text { Analiza el proceso } \\
\text { de rotación de los } \\
\text { empleados a partir } \\
\text { de técnicas } \\
\text { estadisticas }\end{array}$ & $\begin{array}{l}\text { Identifica los } \\
\text { empleados de alto } \\
\text { potencial y establece } \\
\text { planes de desarrollo } \\
\text { especificos }\end{array}$ & $\begin{array}{l}\text { Contempla la } \\
\text { sucesión para los } \\
\text { puestos clave }\end{array}$ \\
\hline $\begin{array}{l}\text { Puestos } \\
\text { clave }\end{array}$ & No se contempla & $\begin{array}{l}\text { Estratifica el análisis } \\
\text { estadistico por } \\
\text { niveles de } \\
\text { complejidad para la } \\
\text { cobertura del puesto, } \\
\text { independientemente } \\
\text { de su carácter } \\
\text { estratégico }\end{array}$ & $\begin{array}{l}\text { Identifica los puestos } \\
\text { clave, según su } \\
\text { influencia en la } \\
\text { creación y } \\
\text { sostenimiento de las } \\
\text { ventajas competitivas }\end{array}$ & $\begin{array}{l}\text { Identifica los } \\
\text { puestos clave } \\
\text { según su influencia } \\
\text { en la creación y } \\
\text { sostenimiento de } \\
\text { las ventajas } \\
\text { competitivas }\end{array}$ \\
\hline Procesos & Todos & $\begin{array}{l}\text { Identificación y } \\
\text { Retención del } \\
\text { talento }\end{array}$ & $\begin{array}{l}\text { Identificación y } \\
\text { Desarrollo del talento }\end{array}$ & $\begin{array}{l}\text { Desarrolloy } \\
\text { retención del } \\
\text { talento }\end{array}$ \\
\hline Autores & $\begin{array}{l}\text { Kristof (1996) y } \\
\text { Lawler, Levenson } \\
\text { \& Boudreau } \\
(2004)\end{array}$ & $\begin{array}{l}\text { Rothwell (1994), } \\
\text { Pascal (2004), } \\
\text { Huselid (2006) y } \\
\text { Kehinde (2012) }\end{array}$ & $\begin{array}{l}\text { Jackson \& Schuller } \\
\text { (1990), Axerold et } \\
\text { al.(2002), Michaels et } \\
\text { al.(2001), Smart } \\
\text { (1999), Ashton \& } \\
\text { Morton (2005), } \\
\text { González, Martinez \& } \\
\text { Pardo del Val (2009), } \\
\text { Tarique \& Schuler } \\
\text { (2010), Sullilion, } \\
\text { Collings \& Calligiuri } \\
\text { (2010), Scullion \& } \\
\text { Collings (2011) }\end{array}$ & $\begin{array}{l}\text { Collings \& Mellahi } \\
\text { (2009) }\end{array}$ \\
\hline
\end{tabular}

Fuente: elaboración propia.

Luego del análisis bibliográfico podemos asumir que cada una de las vías identificadas centra sus esfuerzos de manera específica en uno o varios de los cuatro procesos de la GT: atracción, identificación, desarrollo y retención. Cada vía sigue un enfoque diferente de cada proceso, y analiza su importancia en función del aporte a la organización. Así, hay autores que analizan todos los procesos de la GT por lo que centran sus estudios en la Vía I. Otros analizan en sus trabajos la selección y retención del talento por lo que se pueden identificar con la Vía II. Por su parte, muchos autores se centran en la identificación y el desarrollo del talento, lo que los ubicaría en la Vía III, que es, sin duda, la más seguida en la literatura. Por último, Collings y Mellahi (2009) se centran en el análisis del desarrollo y la retención del talento, es decir, en la Vía IV.

\section{Dimensiones de la GT}

A través del análisis de la literatura hemos podido determinar la existencia de dos dimensiones sobre las que se sostiene un sistema eficaz de GT. La primera de ellas es la dimensión personas. Esta es la dimensión más comúnmente vinculada a la GT, no en vano la palabra Talento tiene una estrecha vinculación con las características de la persona. La GT así concebida entiende a las personas como un recurso que se puede adquirir, moldear y utilizar para ayudar a cumplir los planes de la empresa. Por otro lado, a partir del trabajo de Collings y Mellahi (2009) se hace referencia a la existencia de una segunda dimensión conocida como puestos o posiciones clave de la compañía. Estas son posiciones directamente vinculadas a las ventajas competitivas de la empresa. 


\section{Dimensión relacionada con las personas}

La dimensión "personas" es la más estudiada en el ámbito de la GT. Los procesos para gestionar el talento han ido evolucionando. Hay autores que sólo tratan un proceso como la retención (Kristof, 1996), mientras que otros se refieren a dos, como la atracción y el desarrollo (Axerold et al., 2002; Michaels, Handfield-Jones y Axelrod, 2001; Smart, 1999), o al desarrollo y la retención (Rothwell, 1994; Jackson y Schuller, 1990). Posteriormente, los autores empiezan a incluir tres procesos de la GT (Dries, 2013; Tarique y Schuler, 2010; Collings y Mellahi, 2009; Lewis y Heckman, 2006; Pascal, 2004). Finalmente, trabajos posteriores ya recogen los cuatro procesos (Capelli, 2008; Scullion, Collings y Calligiuri, 2010; Kehinde, 2012).

La atracción del talento se convierte en la primera fase de un plan de GT integral. Las organizaciones deben dotarse de las herramientas necesarias para atraer personal con talento con el que nutrir la organización. Se pueden utilizar diferentes herramientas o explorar diferentes formas de conseguirlo, pero la organización debe mostrarse atractiva para aquellos que buscan un lugar donde desarrollar su potencial (Huselid et al., 2005).

Según Capelli (2008) existen dos formas principales de dotarse de talento a través de la captación externa: 1) contratar talento senior procedente de otras organizaciones, cuyo potencial se estima en función de su historial; 2) hacer contrataciones sistemáticas de un número determinado de personal cualificado con el que ir surtiendo las necesidades de talento de la empresa.

La identificacióndel talento supone tanto la captación del talento externo a través de procesos de reclutamiento y selección, como los procesos específicos para reconocer el talento dentro de la organización (Capelli, 2008; Collings et al., 2009; Scullion et al., 2010; Kehinde, 2012). Esto último se realiza a partir de la evaluación del desempeño y el análisis del potencial de los propios empleados. De una correcta identificación del talento dependerá su posterior desarrollo y la adecuada recolocación de los profesionales.

Por otra parte, si el talento, una vez identificado, no es correctamente desarrollado, puede llevar a que el proceso de GT no sea eficaz (Scullion et al., 2011; Tarique et al., 2010; Collings et al., 2009; Capelli, 2008). Los perfiles profesionales son el fruto de la suma de unas cualidades personales y unas cualificaciones profesionales. Por tanto, el objetivo del desarrollo del talento debe ser sumar a los perfiles personales adecuados, las cualificaciones profesionales requeridas para el tipo de carrera profesional que se pretende. Algunos planes incluyen también el desarrollo personal, con lo que se intenta dotar al sujeto de cualidades personales de las que carece. Esta última parte, sin embargo, es más difícil de desarrollar.

En organizaciones que se dotan de una cantera de talento, estos seleccionados son analizados en profundidad y se extraen las competencias en las que destacan por encima de otros. A la vez se analizan los diferentes campos de desarrollo en los que el individuo puede progresar. Estos campos de desarrollo deben corresponderse al perfil futuro o al plan de carrera que más se asimila al perfil del individuo. De este modo, sus objetivos y los de la empresa se encontrarán correctamente alineados en el largo plazo. Por tanto, para que el desarrollo del individuo sea correcto y se asegure el éxito, debe haber una relación directa entre el campo de interés del individuo y el ámbito en que la empresa quiere recolocarle en el futuro. Con esas dos informaciones se debe realizar un estudio de las competencias profesionales y personales necesarias para el desarrollo futuro de su trabajo y cruzarlo con las competencias que se le han detectado al sujeto durante la fase de identificación.

Por último, la retención del talento asegura la continuidad de los profesionales catalogados como altos potenciales dentro de la empresa. En algunos casos, todo el sentido del plan de GT de la compañía se justifica en la retención de sus mejores profesionales, impidiendo que estos se marchen a la competencia. Por ello, cada vez más, el estudio de los paquetes retributivos se muestra como una herramienta necesaria del proceso. Por un lado, paquetes retributivos demasiado bajos ponen en riesgo todo el sistema de GT, pues pueden provocar una alta rotación de profesionales. Por otro, paquetes retributivos demasiado elevados disminuyen la eficiencia de la empresa haciéndola incurrir en costes elevados por encima de la media sectorial, lo que puede perjudicar sus resultados. 
Cada empresa, a la hora de implantar un programa de GT, da una mayor o menor importancia a cada uno de estos cuatro procesos en función de los resultados que quiera alcanzar. A partir del énfasis dado a cada proceso es posible identificar la vía de GT privilegiada.

\section{Dimensión relacionada con los puestos clave}

Los autores centrados en la parte estratégica de la GT ponen el énfasis en la identificación de las posiciones clave (Boudreau y Ramstad, 2005b; Collings y Mellahi, 2009). Estos modelos se encuentran con la dificultad de cómo delimitar su impacto real en la estrategia de la empresa a la hora de definir las posiciones clave.

Collings y Mellahi (2009) establecen que para identificar las posiciones clave es preciso reconocer primero la fuente de ventajas competitivas. Existe una relación directa entre las ventajas competitivas y las competencias clave, de modo que, para que existan ventajas competitivas sostenibles es necesaria la existencia de competencias clave. Por lo tanto, entendemos que la GT desde el enfoque estratégico, debe analizar los puestos clave que forman parte de esas competencias. Esto debe permitir, por medio de las personas que ostentan esos cargos, la sostenibilidad y mejora a largo plazo de las ventajas competitivas (Collings y Mellahi, 2009).

El objetivo final del proceso es el de encontrar cuáles de esas posiciones clave poseen verdadera importancia estratégica y, por tanto, deben ser especialmente tratadas por el plan de GT.

\section{Relación entre las vías de estudio y las dimensiones clave de la GT}

La relación entre las vías de estudio y las dimensiones clave se puede definir a partir de un modelo matricial sencillo. Los ejes quedan establecidos en función de la importancia relativa que el plan de GT otorga a cada una de las dimensiones (puestos clave: eje horizontal; personas: eje vertical). A partir de ahí se establecen las cuatro regiones correspondientes a cada una de las vías de estudio (figura 1).

De esa manera, la Vía I en la que las personas centraban el enfoque de la GT ocuparía la posición superior izquierda. La Vía II, que concede la importancia a ambas dimensiones tan solo como mera aportación estadística, se encontraría en la parte inferior izquierda de la matriz. La Vía IV, claramente enfocada a la dimensión puestos clave, descrita por Collings y Mellahi (2009), ocuparía la parte inferior derecha. 


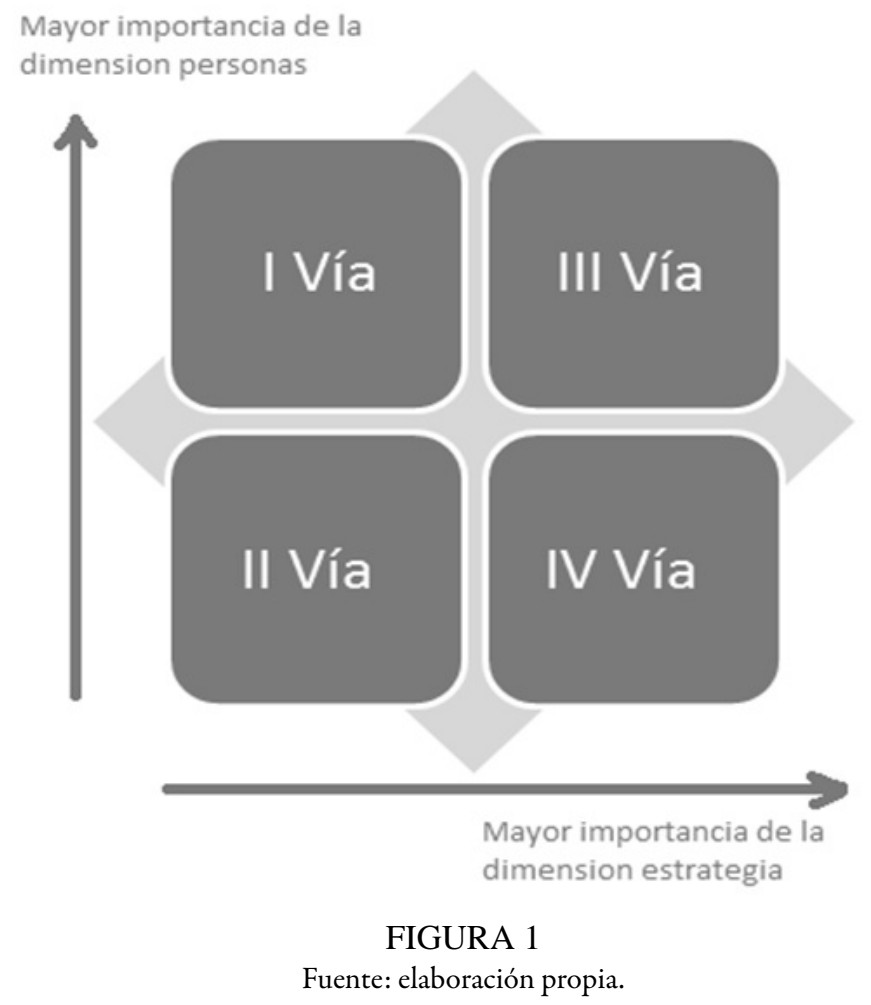

Por último, la Vía III, quedaría situada en la parte superior derecha, como consecuencia de la atención simultánea a la dimensión personas y la dimensión puestos clave. En esta vía se contempla un uso intensivo de ambas dimensiones para proporcionar a la organización los mejores individuos en las posiciones clave. Además, el trabajo de ambas dimensiones se realiza en paralelo de forma coordinada.

Este modelo puede ser de utilidad a la hora de clasificar en una primera instancia a las empresas de acuerdo con su comportamiento en el proceso de diseño del plan de GT. Esto nos lleva a plantear dos cuestiones de investigación:

Q1. ¿Coexisten cuatro vías diferentes de gestionar el talento en las empresas?

Q2. ¿Existe relación entre las dimensiones y los procesos de la GT con la vía de enfoque?

Para dar respuesta a estas dos cuestiones de investigación, la metodología utilizada es el estudio de casos (Yin, 1994).

\section{Metodología}

\section{El estudio de casos como metodología de investigación}

El estudio de casos es un método de investigación que puede contribuir a la construcción, mejora y desarrollo de perspectivas teóricas rigurosas en torno a las empresas y organizaciones (Bonache, 1999; Eisenhardt, 1989; Yin, 1998). Este método permite un estudio de los procesos en su propio contexto, además de análisis longitudinales de las acciones y significados que tienen lugar y son construidos en las organizaciones (Eisenhardt, 1989; Hartley, 1994). Igualmente, la naturaleza abierta de la toma de datos permite que los procesos sean analizados con una considerable profundidad, a diferencia de lo que sucede con el empleo 
de otras metodologías de investigación. Siguiendo a Yin (1994), el estudio de casos permite analizar, en el contexto actual empresarial, porqué y cómo gestionar el talento, es decir, si existen diferentes vías de enfoque.

Para este estudio, optamos metodológicamente por el estudio de casos por los siguientes motivos: (i) la carencia de estudios sobre la GT tanto a nivel mundial como regional (Al Ariss, Cascio y Paauwe, 2014); (ii) porque implica un cambio en la estrategia corporativa de las empresas, debido a factores externos e internos de reciente aparición en el paradigma empresarial (Festing y Schäefer, 2014); y (iii) porque existe poca experiencia y base teórica disponible (Lewis y Heckman, 2006; Nijs et al., 2014). Además, según Eisenhardt (1989), esta metodología es recomendable para la investigación de temas muy novedosos, sobre los que se desea avanzar en la teoría. Tal es el caso que plantea la GT, definida por algunos como una disciplina en una etapa adolescente y en crecimiento (Thunnissen, Boselie y Fruytier, 2013; Collings, Scullion y Vaiman, 2015; Gallardo et al., 2015).

Nuestra investigación puede ser clasificada en los siguientes términos. Primero, va a tener una naturaleza explicativa, puesto que, a partir de las cuestiones de investigación planteadas y en función del marco conceptual obtenido de la revisión de la literatura, se pretenden encontrar evidencias empíricas que permitan avanzar en la discusión en la teoría. Segundo, en cuanto al número de casos, se trata de un estudio de casos múltiples. A partir de la realización de un estudio de caso piloto, decidimos replicar el proceso investigador en otras tres empresas españolas que han implantado un proceso de GT. Así, a partir de la lógica de la réplica, poder extraer conclusiones de la investigación. Para nuestro estudio, la elección de un único caso no sería recomendable, puesto que no permite realizar generalizaciones, limitándose a ser un estudio descriptivo y parcial en sus conclusiones. Para incrementar la validez externa y reducir la parcialidad, hemos decidido replicar el proceso investigador en cuatro empresas. En este sentido, seguimos a Eisenhardt (1989) que recomienda este número de casos para no perder el control de los datos, ni generar saturación de información con un número mayor.

\section{Selección de casos}

Por razones de confidencialidad identificamos las empresas como los casos A, B, C y D. Estas cuatro empresas han sido seleccionadas por su capacidad de transferibilidad o generalización analítica, ya que han implantado un programa de GT. El Caso A es una empresa fabricante de aviones y equipos aeroespaciales a nivel global. El Caso B es una corporación europea de servicios de tecnologías de la información, líder global en transformación digital. El Caso $\mathrm{C}$ es un grupo global e integrado referente internacional en el sector energético. Finalmente, el Caso D es una empresa española dedicada al diseño, venta y distribución de dispositivos digitales.

\section{Recopilación y análisis de datos}

En nuestra investigación se han empleado múltiples fuentes de evidencia que fueron debidamente trianguladas. Estas son:

a) Evidencia documental. Se examinaron documentos internos como memorias, informes, estudios internos, páginas web, archivos de presentaciones, archivos de imagen y sonido; y externos como publicaciones especializadas, informes de organismos y medios de comunicación. Se aprovecharon así las ventajas que proporcionan las nuevas tecnologías de la información, gracias a los recursos proporcionados por internet y la web corporativa de las empresas para la obtención de información adicional a las antes mencionadas. 
b) Observación directa. Visita guiada a la propia empresa, la cual nos ha permitido entrar en contacto con la organización y la obtención de notas de campo que aumentarán la validez de los resultados obtenidos a través de otras fuentes. Las notas recopiladas han sido cuidadosamente clasificadas. Todas ellas están catalogadas tomando como criterio una frase descriptiva que recoge el contenido genérico de la misma. Igualmente, se han especificado en ellas la hora, día, lugar y la persona que ha proporcionado la información. Ésta se ha recogido en el mismo día que se ha obtenido para evitar lapsus temporales. También se ha asistido a conferencias de los directivos, impartidas tanto en ámbitos académicos como profesionales.

c) Cuestionario. Se diseñó un cuestionario integrado por preguntas abiertas, preguntas dicotómicas, preguntas categóricas de respuesta sugerida y preguntas cerradas de entre 4 y 12 ítems cada una. Fueron medidas con una escala de Likert de grado 5. Estas cuestiones planteaban preguntas relacionadas con la GT y con la atracción, identificación, desarrollo y retención del mismo. La escasez de investigaciones empíricas en el campo de la GT, así como la inexistencia de un cuestionario validado en la literatura, nos llevó a diseñar este cuestionario inspirándonos en la literatura previa. Se realizó una validación previa del instrumento, testándolo con un profesional de empresa experto en recursos humanos y tres académicos del área. Para ello fue utilizado el caso piloto. El cuestionario fue revisado personalmente durante las entrevistas para asegurar la comprensión de todas las cuestiones tratadas y, posteriormente, enviado por email a las personas consideradas clave en el proceso de GT de las empresas analizadas. Se obtuvieron cuestionarios cumplimentados de dos informantes clave por su vinculación con la GT en los cuatro casos analizados. Las principales variables, dimensiones e indicadores recogidos en el cuestionario se presentan en el Anexo.

d) Entrevistas. Se realizaron un total de 19 entrevistas con varios directivos implicados directamente en la gestión de recursos humanos de las empresas y/o en la GT. Para reducir el sesgo en las respuestas obtenidas, en todos los casos se ha entrevistado a más de un informante. En el caso A, las personas entrevistadas fueron la directora de GT (tres entrevistas) y el director del área de formación (dos entrevistas). En el caso B, se entrevistó al director de recursos humanos de una unidad de negocio (una entrevista), a la directora de la GT (tres entrevistas) y al director de innovación de procesos (dos entrevistas). En el caso C, se entrevistó a la directora de liderazgo (tres entrevistas) y a la directora de innovación y talento (dos entrevistas). En el caso D, fueron entrevistados el director general (una entrevista) y el director de recursos humanos (dos entrevistas). Las entrevistas se llevaron a cabo en el periodo septiembre de 2016 - marzo de 2017, excepto en el caso piloto, cuyas primeras entrevistas tuvieron lugar en octubre de 2015.

La ficha técnica de la investigación se recoge en la tabla 2. 
TABLA 2

\begin{tabular}{|l|l|}
\hline \multicolumn{2}{|c|}{ FICHA TÉCNICA DE LA INVESTIGACIÓN } \\
\hline $\begin{array}{l}\text { Propósito de la } \\
\text { investigación }\end{array}$ & $\begin{array}{l}\text { Analizar la existencia de 4 enfoques o vias diferenciados de la gestión del } \\
\text { talento. } \\
\text { Analizar las dimensiones: personas y puestos clave de la gestión del talento } \\
\text { Relacionar las dimensiones y los procesos claves de la gestión del talento con } \\
\text { las 4 vias de enfoque. }\end{array}$ \\
\hline $\begin{array}{l}\text { Metodologia de } \\
\text { investigación }\end{array}$ & $\begin{array}{l}\text { Estudio de casos contemporáneo múltiple de carácter holistico (unidad de } \\
\text { análisis simple). Estudio exploratorio, descriptivo y explicativo. }\end{array}$ \\
\hline Tipo de muestra & $\begin{array}{l}\text { Muestra lógica y teórica (capacidad de generalización analitica del fenómeno } \\
\text { estudiado- transferibilidad), no de forma aleatoria (muestreo y generalización } \\
\text { estadistica). }\end{array}$ \\
\hline $\begin{array}{l}\text { Muestra y Unidad } \\
\text { de análisis }\end{array}$ & $\begin{array}{l}\text { Cuatro empresas que operan en España y han implantado un programa de } \\
\text { gestión de talento. }\end{array}$ \\
\hline $\begin{array}{l}\text { Métodos de } \\
\text { recogida de la } \\
\text { evidencia }\end{array}$ & $\begin{array}{l}\text { Evidencia documental } \\
\text { Observación directa } \\
\text { Entrevistas } \\
\text { Cuestionarios }\end{array}$ \\
\hline $\begin{array}{l}\text { Fuentes de } \\
\text { información }\end{array}$ & $\begin{array}{l}\text { Interna: documentación (memorias, informes y estudios internos), archivos } \\
\text { (páginas webs, archivo de presentaciones, archivos de imagen y sonido), } \\
\text { entrevistas en profundidad, cuestionarios, contexto fisico real. } \\
\text { Externa: publicaciones especializadas, bases de datos, informes de } \\
\text { organismos oficiales y medios de comunicación. }\end{array}$ \\
\hline Informantes clave & $\begin{array}{l}\text { Directivos de las empresas de la muestra (dos de cada caso) con participación } \\
\text { activa y directa en el proceso de Gestión del talento }\end{array}$ \\
\hline $\begin{array}{l}\text { Métodos de } \\
\text { análisis de la } \\
\text { evidencia }\end{array}$ & Fundamentalmente de tipo cualitativo \\
\hline $\begin{array}{l}\text { Enfoque } \\
\text { cientifico }\end{array}$ & $\begin{array}{l}\text { Inducción analitica (generalización analitica) } \\
\text { Procesos deductivos (proposiciones teóricas) }\end{array}$ \\
\hline $\begin{array}{l}\text { Evaluación del } \\
\text { rigor y calidad } \\
\text { metodológica }\end{array}$ & $\begin{array}{l}\text { Validez (constructiva, interna y externa), fiabilidad, consistencia (teórico- } \\
\text { interpretativa y contextual) }\end{array}$ \\
\hline $\begin{array}{l}\text { Fecha de } \\
\text { realización }\end{array}$ & Septiembre 2016- Marzo 2017 \\
\hline
\end{tabular}

Fuente: elaboración propia.

Respecto al análisis de datos, este estudio adopta un enfoque descriptivo, exploratorio y explicativo, para evaluar si existen dos dimensiones para la GT que conduce a las empresas a implantar el proceso de manera diferenciada. Para ello, se llevó a cabo un análisis de contenido (Bardin, 1991; Easterby-Smith, Thorpe y Jackson, 2012), utilizando el software gratuito VosViewer (versión 1.6.5.) para analizar contenidos específicos de las entrevistas. Esta herramienta crea un mapa basado en datos de texto, creando co-ocurrencias de términos. Los mapas de términos pueden ser creados directamente a partir de un cuerpo de texto.

Las entrevistas, que tenían una duración media de 90 minutos, fueron transcritas, revisadas y guardadas en el formato "texto sin formato". A partir de aquí, el análisis de datos permitió identificar clústeres que recogían palabras clave, las cuales pudimos relacionar con algunas de las cuestiones de investigación. El análisis de contenido fue especialmente útil, ya que permitió reproducir y volver analizar de forma comparada los datos obtenidos sistemáticamente, además de construir una base de datos.

Si bien se considera que la presente ha sido una estrategia de investigación idónea por la novedad del tema y la escasez de trabajos empíricos específicos, el método posee algunas limitaciones metodológicas como son los problemas de validez, fiabilidad y consistencia asociados con el sesgo introducido por el investigador. Dichas limitantes se han tratado de gestionar correctamente. Para garantizar el rigor de la investigación, Bonache (1999) propone aplicar diferentes tácticas o pruebas como son: (1) La validez constructiva a través de la triangulación, la cual permite una convergencia y coherencia entre los resultados obtenidos y el rigor y análisis de los datos. En nuestro estudio se logra la triangulación a través del uso de múltiples fuentes de evidencias que, analizadas de forma conjunta, nos lleva a alcanzar coherencia de los resultados; (2) La validez externa, justificada a través de la selección de más de un caso, lo que ayuda a verificar a través de la réplica las conclusiones extraídas. De este modo, se incrementa la posibilidad de generalización analítica, especialmente cuando dicha selección se ha llevado a cabo por criterios teóricos, como es el caso de nuestro estudio. (3) la validez interna que se fundamenta en el carácter cíclico de los casos que se han ido desarrollando en la investigación. 


\section{Resultados y Discusión}

El objetivo del trabajo era analizar si coexisten las cuatro vías de estudio de la GT y, analizar la relación entre estas cuatro vías con sus dimensiones y procesos. De las evidencias encontradas se concluye que cada empresa articula la GT como un conjunto de prácticas y procesos específicos, de forma que, no siempre se enfoca en las mismas dimensiones, ni se otorga la misma importancia relativa a cada uno de los procesos.

A continuación, se analizan las evidencias de los cuatro casos para poder determinar si se enfocan o no en una vía de estudio de la GT. Así, para el Caso A se especifica que "la GT está centrada en las personas, siendo el principal objetivo de esta la rotación interna de empleados dentro de la compañía con independencia del puesto". Por ello, se pone énfasis en la dimensión "personas". Por otro lado, respecto a los diferentes procesos y a la atracción, la directora de GT (Talent Manager) afirma: "no tenemos problemas de atracción del talento ya que la empresa es considerada líder del sector y posee muy buena imagen como empleador" por lo que no es un proceso que se considere esencial. Sin embargo, la empresa implanta políticas innovadoras de identificación de talento interno, como por ejemplo la nueva practica "Hidden Treasures" (Tesoros Escondidos). Este mecanismo permite, a través de actividades de bases de datos internas, identificar personal con unas determinadas características o potencial, que aun sin haber sido identificados nunca por sus superiores como posibles talentos, reúnen requisitos que son valorados por la compañía. Esta herramienta, además, se refuerza con la utilización de complejos procesos de assessment center (centros específicos de evaluación en donde se hacen entrevistas), Business Cases (estudios de casos de negocios) y Stakeholders Meeting (encuentro con grupos de interés). Estos hallazgos encuentran apoyo en la literatura revisada, como en Lehman et al. (2011), que apostaban por el assessment center como un instrumento clave para la identificación del talento, o en Garavan, Carbery y Rock (2012), que defienden novedosas herramientas de networking como los eventos de visibilidad para desarrollar el talento.

En cuanto al desarrollo del talento, para el caso A, debe ser el empleado quién dirija su desarrollo, eligiendo qué formación es la más adecuada para su perfil y el plan de carreras que quiere seguir. Por otra parte, no es subvencionado ningún tipo de máster o curso de especialización externo a la empresa. En este aspecto, según la directora de GT "se trata de pasar a un modelo proactivo en que los empleados sean los que pasen a la iniciativa en su desarrollo y la generación de sus oportunidades. La empresa le da las herramientas para que pueda serlo". Por lo que no es el proceso clave para la GT.

Por último, el caso A basa sus programas de retención en aportar "a los empleados un entorno de trabajo inigualable con oportunidades internacionales, con motivación constante a los empleados y a través de la existencia de políticas de conciliación punteras a nivel internacional" (Talent Manager). De las evidencias de los cuestionarios y las entrevistas se deduce que los empleados de esta empresa disfrutan de salarios superiores a la media del sector en los diferentes países donde la empresa está presente, además de, en general, tener jornadas laborales más reducidas que la competencia y más días de vacaciones. El hecho de hacer de la retribución una herramienta de retención del talento, está en línea con lo propuesto por Rynes et al. (2005) y Gunter et al. (2012). Además, en el éxito de los programas internos de retención, la compañía considera que es de gran utilidad la existencia de entrevistas directas con los candidatos donde se enfoca su perfil y su futuro, y se realiza la gestión de las expectativas de los talentos. Estas entrevistas, tratan de alinear constantemente las necesidades o motivaciones de los talentos con las de la empresa.

Por todo ello, el caso A le da mucha importancia a este proceso y nos lleva a concluir que los procesos más importantes para esta empresa para gestionar su talento son la identificación y la retención. Este hecho justifica la mayor importancia relativa otorgada a la dimensión personas.

Por su parte, en el Caso B se considera que las dimensiones personas y puestos clave son importantes a la hora de implantar el programa, en línea con lo defendido por Collings y Mellahi (2009). En este caso se presta mucha atención a las fases de identificación y desarrollo. Respecto a la identificación, llevan a cabo un exhaustivo proceso para identificar el talento, en el que participan varias áreas de la compañía. Para el 
desarrollo del talento, implementan un programa específico y personalizado a cada miembro del talent pool (agrupaciones de talento) identificado previamente, por lo que existen diferentes programas de desarrollo en función del perfil interno. Por ejemplo, posee un programa específico de senior talent management denominado Gold. El programa Gold se realiza en conjunto con prestigiosas entidades de educación superior europea. Este desarrollo se complementa, en ocasiones, con formación externa muy costosa, tal como propone Garavan et al. (2012).

Respecto a la retención cabe destacar que, según se recoge en la entrevista realizada a la directora de talento, "Ser talento no está directamente relacionado con la posibilidad de acceder a políticas retributivas superiores a la media para la posición ni diferentes o específicas medidas de flexibilidad o conciliación”. La política retributiva responde a niveles salariales previamente preestablecidos en una empresa con gran cantidad de trabajadores, por lo que no es un proceso clave de GT.

En este caso, se observa un mayor peso en los procesos de identificación y desarrollo que en el resto de áreas, lo cual es coherente con el enfoque doble sobre personas y puestos clave. Se considera el factor humano una variable clave tanto en la implantación como en la formulación de estrategias, como defienden Alonso y Garcia Muiña (2014).

En el Caso C, la dimensión puesto adquiere una gran relevancia para la GT y, por ello, han creado un departamento específico de GT. Según el cuestionario, se obtiene el objetivo que se persigue con este programa: conseguir mejores resultados tanto individuales como organizativos y mejorar la posición estratégica de la compañía. La importancia de los puestos clave como dimensión de la GT se refleja de la siguiente forma: "El talento son las personas, son los que tienen las competencias que tienen que poner a disposición de la empresa. Sin embargo, va en paralelo con los puestos clave. No tendría sentido estar desarrollando un talento que la empresa no va a necesitar. Podríamos lograr una empresa con mucho talento, pero si no está orientado al negocio no llevaría a ninguna parte" (Responsable de innovación y del talento).

Respecto a los procesos identificados como clave son el desarrollo y la retención. La confianza en el desarrollo interno y el hecho de aparecer de forma asidua en las primeras posiciones de rankings de employer branding (buen empleador) hace que tengan un índice de rotación muy bajo. Esto permite que la atracción no sea un proceso esencial (exceptuando la captación del talento joven). Igual ocurre con la identificación, para la que utilizan, de modo general con todos los empleados, una herramienta de evaluación del desempeño llamada People review. Sin embargo, para el desarrollo del talento emplean planes de desarrollo, individuales o grupales específicos para diferentes colectivos (talent pool). Esto es complementado con novedosas herramientas de networking, como eventos de visibilidad (desayunos, reuniones, comités) y con formación premium, en línea con lo que proponen Garavan et al. (2012). Además, se asignan proyectos transversales o clave para completar el desarrollo del talento. En este sentido, se considera que las prácticas clásicas de gestión de recursos humanos para los empleados clave podrían ser insuficientes, debiendo ser sustituidas por otras más innovadoras y completas como employer branding, assessment center, mentoring o coaching. Tal como afirman Kong, Chadee y Raman (2013), estas prácticas permitan desarrollar otras capacidades más allá del conocimiento y el aprendizaje como la capacidad de adaptación al cambio, la capacidad de liderazgo, etc.

Dentro de los empleados identificados como potenciales para desarrollar su talento, en el Caso C, se identifican aquellos a los que se les asignan carreras Fast Track. Se busca en ellos un desarrollo acelerado de las capacidades del potencial. Por último, en cuanto a la retención, cabe destacar una extraordinaria capacidad de retención de profesionales de la compañía, ya que cuenta con un sistema retributivo muy competitivo. El caso C, por tanto, presta mayor atención a los procesos de desarrollo y retención, centrándose en la dimensión puestos clave de la GT.

Por último, para el Caso D, la GT se centra en las personas, siendo la fase de atracción del talento la que hasta el momento está más desarrollada, tal como se recoge tanto en el cuestionario como en la entrevista al director de recursos humanos. Para la atracción, existen planes de selección que pretenden atraer a profesionales con talento a través de actividades de employeer branding, tratando de mostrarse como 
una empresa atractiva en la que trabajar. También destaca su Cátedra, firmada con una universidad pública española. Se trata de un proyecto formativo-evolutivo, transversal, que va desde la impartición de seminarios y cursos a la colaboración en programas de $\mathrm{I}+\mathrm{D}+\mathrm{i}$ con la Universidad. En este marco, la empresa desarrolla becas, programas de colaboración con la Universidad y estrategias de employeer branding.

Respecto a la identificación del talento, el Caso D lleva actualmente este proceso a través de un sistema informal de comunicación, siendo realizado, por tanto, mediante criterios exclusivamente subjetivos. Para el desarrollo del talento la empresa aplica programas de formación clásica que se desarrollan tanto interna como externamente. Según su director general, estos programas "Se centran sobre todo en potenciar las dimensiones del talento que son más débiles en el individuo". En cuanto a la retención de estos profesionales, la empresa pone especial interés en retribuir con compensaciones superiores a la media a sus mejores empleados, así como en buscar la motivación de los empleados considerados talento. En definitiva, el caso D presta una mayor atención al proceso de atracción de talento, lo que se justifica por estar al inicio del proceso de implantación.

En la tabla 3 se resumen las dimensiones y los principales procesos de GT de los cuatro casos analizados, así como la vía de enfoque a la que se aproximan.

\section{TABLA 3}

\begin{tabular}{|l|l|c|c|c|c|}
\hline \multicolumn{2}{|l|}{} & CASO A & CASO B & CASO C & CASO D \\
\hline $\begin{array}{l}\text { Dimensiones } \\
\text { de la } \\
\begin{array}{l}\text { Gestión del } \\
\text { Talento }\end{array}\end{array}$ & Personas & & & & \\
\hline Puestos clave & & & & \\
\hline Proceso atracción & & & & \\
\hline Proceso identificación & & & & \\
\hline Proceso desarrollo & & & & \\
\hline Proceso retención & & & & \\
\hline Vía de Gestión de Talento & II & III & IV & I \\
\hline
\end{tabular}

Nota: celda negra significa relación fuerte entre esas dos dimensiones; celda gris: relación media y celda blanca: ausencia de relación). Fuente: elaboración propia.

Una vez analizada las evidencias de cada empresa, pasamos a desarrollar el estudio comparado para cada proceso de GT. Así, por ejemplo, en los casos A, B y D la atracción del talento se desarrolla mediante planes específicos de selección de profesionales. Son utilizadas herramientas como el employeer branding, acuerdos con universidades o becas formativas, que hacen que esta fase sea un proceso relevante en su modelo. En el caso C, la atracción de profesionales, sin ser tan relevante, pretende cubrir una necesidad diferente, pues la búsqueda se centra en perfiles junior, que acaben de terminar sus estudios superiores en materias esenciales para la empresa. Nuestros hallazgos encuentran apoyo en la literatura revisada. Con respecto a la etapa de atracción, algunos trabajos previos como los de Knox y Freeman (2006), Martin, Gollan y Grigg (2011), Kucherov y Zavyalova (2012) y Hanif y Yunfei (2013) ya proponían el employer branding como una herramienta clave para atraer el talento. Igualmente, Gandz (2006) ya señalaba en su trabajo cómo la reputación actuaba como imán para atraer talento.

La fase de identificación se revela como un proceso clave en los casos A, B y C, en donde para identificar a los empleados considerados como talento se utilizan, principalmente, las evaluaciones de rendimiento anuales o semestrales, pero también para poder identificar el potencial futuro se llevan a cabo comités o reuniones de expertos internos. En el caso D esta fase es mucho menos significativa e incluso, la incorporación de los empleados considerados como talento a los diferentes talent pool, se realiza de modo informal, sin acometer una evaluación de rendimiento cíclica.

Por tanto, en nuestros resultados sobre la fase de identificación, encontramos que ésta se efectúa principalmente en las evaluaciones de rendimiento formales.

Estos resultados son respaldados por la literatura. En la etapa de identificación, Frank, Finnegan y Taylor (2004) y Björkman y Smale (2010) hacen referencia a la evaluación del desempeño como práctica para identificar el talento, mientras que Nijs et al. (2014) se centra en la evaluación del potencial. 
Debe destacarse que el desarrollo de los empleados identificados como talento, es una de las áreas más trabajadas por las cuatro empresas. En los casos C, A y B, se aplican herramientas específicas de desarrollo como el Mentoring o el Coaching. Otras herramientas, como la formación interna y externa, la movilidad interna y la rotación por departamentos, son utilizada por todas las empresas analizadas. Todas estas herramientas se encuentran en la literatura. En relación con la etapa de desarrollo, Garavan et al. (2012) y Gannon y Maher (2012) consideran el Mentoring para el desarrollo del talento, mientras que Joo, Sushko y Mclean (2012) incorporan a éste el Coaching.

Por último, la fase de retención, que es esencial para los casos A y $\mathrm{C}$, se revela menos significativa para los casos $\mathrm{B}$ y D. Las empresas de los casos A y C poseen políticas retributivas que permiten evitar la compresión salarial entre los empleados talentosos y valorar, de esa forma, rendimientos y desempeños superiores a la media. Estas son las empresas que muestran mayor diversificación de prácticas de retención. Las evidencias sugieren que ambas compañías son las que tienen menor nivel de rotación no deseada de trabajadores con talento. Dentro de la literatura, Rynes et al. (2005) y Gunter et al. (2012) proponen la retribución como herramienta de retención del talento.

La importancia que se da a cada uno de los procesos varía según el caso, encontrando en cada empresa diferentes conjuntos de prácticas. El caso A demuestra tener más actividad en las fases de Identificación y Retención. El caso C, por su parte, muestra mayor peso en las fases de Desarrollo y Retención. Ya el caso B muestra fortalezas en los procesos de Identificación y Desarrollo, pero también debilidades en Atracción y Retención. Finalmente, el Caso D muestra un menor desarrollo general en todas las fases que el resto de empresas, pero mayor fortaleza en la fase de Atracción. Este último resultado encaja con las necesidades de la empresa que, involucrada durante años en un proceso de expansión, ha centrado sus esfuerzos en la necesidad urgente de cubrir nuevas posiciones, privilegiando la atracción por encima de las demás áreas.

En relación con la dimensión "puestos clave", se encuentra que sólo los casos B y C tienen como prioridad la identificación de los puestos clave de la empresa, según los procesos de GT que defienden Collings y Mellahi (2009).

Según las prácticas de GT llevadas a cabo por las empresas y los diferentes pesos obtenidos en las diferentes fases, se puede identificar su actividad dentro de una de las cuatro vías propuestas por la literatura académica (Figura 2).

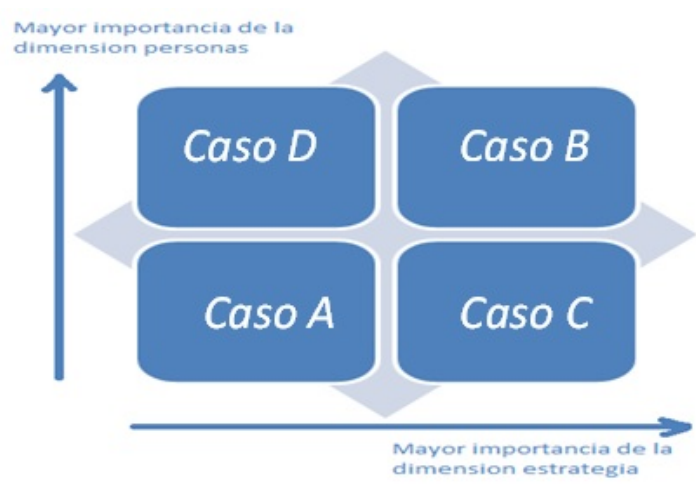

FIGURA 2

Vías de Gestión del Talento seguidas por las empresas analizadas en el estudio de casos Fuente: elaboración propia.

El caso A, con mayor peso en Identificación y Retención, puede ser un ejemplo de la Vía II de estudio. $\mathrm{Al}$ respecto, Becker y Huselid (2006) sugería esta alternativa para empresas con empleo intensivo de mano de obra muy cualificada. Además, en este caso la GT se asemeja a un sistema clásico de planificación de la sucesión (Jackson y Schuler, 1990; Rothwell, 1994) y no se detectan las características que debe tener para ser considerado un programa específico y estratégico para la empresa. 
El caso B muestra un mayor peso en Identificación y Desarrollo que en el resto de áreas, pudiéndose asimilar a la Vía III de trabajo, donde el talento se identifica con los individuos que muestran un alto rendimiento y potencial. Por tanto, la GT tiene un carácter exclusivo, dirigido a determinadas personas claramente identificadas, que desempeñan roles diversos en la organización y que implican un elevado valor añadido, como defienden Axelrod et al. (2002).

El caso C, con un mayor peso en Desarrollo y Retención, puede ser catalogado dentro de la Vía IV propuesta por Collings y Mellahi (2009). En este caso, por tanto, se da más énfasis a las posiciones clave y a las necesidades específicas de las mismas, por lo que la GT pretende asegurar el mantenimiento de las ventajas competitivas a largo plazo garantizando el reemplazo adecuado en dichos puestos clave.

Por último, en el caso de D, los objetivos por los que implantaron el programa de GT fueron principalmente la captación de talento joven. Esto debido al hecho de tratarse de una empresa en creciente expansión y que necesitaba nutrirse rápidamente de este tipo de profesionales. Además, se vinculó directamente con el departamento de RRHH, por lo que la GT se puede entender como una clásica dirección estratégica de los recursos humanos. En este sentido, su director de RRHH afirma: "La GT sería el área 'Hard' en la que se desarrolla la aportación de valor de recursos humanos a la empresa a través de la valorización de las personas". Por lo que, en este caso, la GT no deja de ser una nueva forma renombrar la gestión de los recursos humanos, por lo que no aplicaría la máxima defendida por Chuai Preece y Iles (2008, p. 908): la GT "no debería ser simplemente considerada como vino viejo en botellas nuevas".

\section{Conclusiones}

La GT constituye un área de investigación en proceso de desarrollo caracterizada aún por la ambigüedad de muchos de sus conceptos. La necesidad de conocer si coexisten en la realidad empresarial las cuatro vías de enfoque de la GT, así como sus dimensiones y procesos clave, han justificado el desarrollo del trabajo.

El presente trabajo ha contribuido, en ese sentido, al análisis de la literatura previa sobre las diferentes vías de estudio de la GT. Esto ha sido complementado a través de un estudio de casos realizado en el ámbito empresarial. La metodología del estudio de casos es adecuada para la investigación de temas muy novedosos sobre los que se desea avanzar en la teoría, como es este caso (Eisenhardt, 1989). Esto permite enriquecer una disciplina dominada por trabajos teóricos, especialmente evidente en el caso español (Gallardo-Gallardo et al., 2015).

Junto a estas implicaciones académicas, el presente estudio tiene otras de carácter directivo o de soporte a la toma de decisiones, además de su posible influencia en los empleados considerados como talento. De este modo, planteamos la necesidad de que algunas decisiones de GT deban ser reformuladas por las compañías.

Por ejemplo, en el caso C, cabe destacar dos cuestiones: en primer lugar, a pesar de que la GT gira en torno a la dimensión puestos clave -otorgando una notable relevancia a su papel estratégico-, jerárquicamente se hace depender del departamento de recursos humanos. Esto puede suponer un notable sesgo a la hora de identificar y evaluar las principales fuentes de ventaja competitiva de la compañía, en línea con lo defendido por Colling y Mellahi (2009). Además, la forma en que el caso C define lo que entiende por GT resulta incoherente con la vía que le da soporte (Vía IV). Mientras que esta vía se apoya en la dimensión puestos clave, en el caso C la importancia de la GT recae en las personas.

En el caso B, por su parte, se eleva la responsabilidad jerárquica de la GT a unidades organizativas estrechamente vinculadas con la dirección general de la empresa. Por ello, en este caso, la decisión sobre donde ubicar organizativamente la GT resulta mucho más coherente con su función estratégica. Igualmente, se concede igual importancia tanto a la dimensión personas como a la dimensión puestos clave, por lo que tiene coherencia con la Vía III, en la que se apoya. 
Por otro lado, se observa que, en determinadas circunstancias, no son coherentes lo objetivos, las estrategias y las acciones de GT. Así, en el caso B, se pone un gran énfasis en la reducción de la rotación no deseada, sin embargo, se dedican pocos recursos al proceso de retención de talento. Es incongruente que la empresa implante la GT con el objetivo de reducir la rotación de los empleados, y no se preste atención a las políticas o herramientas de retención del talento, como lo hacen otras compañías. En este sentido, los empleados que formen parte del programa de GT no verán recompensado el esfuerzo que realizan por desarrollarse y crecer dentro de la compañía.

Para el caso A, por su parte, la GT trata de ser un proceso dinámico con características que tratan de ser diferenciadoras, pero está centrado en las personas y no en los puestos clave. En este sentido, la GT se asemeja a un sistema clásico de planificación de la sucesión. Aunque parece seguir la Vía II, no se detectan características de un programa exclusivo que lleve a diferenciar este proceso y demuestre así su carácter estratégico. Además, se considera un proceso reversible de forma que el empleado identificado como talento tiene que estar motivado y tiene que querer formar parte del proceso. En el caso de éste disminuya el desempeño y pierda la motivación, dejará de pertenecer al programa de desarrollo del talento. Por otro lado, puede crear desmotivación en los empleados no integrados en el programa, los cuales pueden no llegar a comprender tal decisión.

Por último, tanto por los objetivos de implantación del proceso como por su vinculación específica con la gestión de los RRHH, en el caso D, la GT se puede entender como una forma más avanzada de gestionar a los empleados, pero no se vincula a un programa específico de desarrollo del talento ni a los puestos clave de la compañía.

En resumen, en ciertas empresas puede llegar a confundir el hecho de la GT no posea aun una visión estratégica unificada, con un vínculo real entre el talento y el logro de ventajas competitivas. En este sentido, no es claro para dichas compañías si las prácticas llevadas a cabo son diferenciadoras o no respecto a la gestión tradicional de recursos humanos. Es esta la razón por la que aún se mantiene esta clasificación de la GT en cuatro vías.

Podemos concluir que las empresas implantan la GT de manera diferenciada: unas prestando más atención a la dimensión personas y otras centrándose en el análisis de los puestos clave. Además, unas prestan más atención a procesos de atracción y retención del talento humano, mientras que otras hacen hincapié en la necesidad de identificar y desarrollar el talento interno de la compañía.

Sin embargo, independientemente de cómo sea implantada, la GT no sólo tiene beneficios para las empresas sino también para sus empleados. A través de este proceso se incrementan sus conocimientos y habilidades, al mismo tiempo que se le brindan las herramientas necesarias para desarrollar su talento y crecer en la compañía. Así, se logra un mayor ajuste entre el empleado y la empresa, que es uno de los retos esenciales a los que se enfrentan las compañías del siglo XXI. Un programa específico de GT permitirá poder alinear los objetivos individuales de los empleados con los objetivos corporativos y la estrategia empresarial. De este modo, se mejorará la comunicación y retroalimentación con el empleado, quien estará más motivado al percibir que se le incentiva de una manera más justa de acuerdo con el esfuerzo realizado. Como consecuencia, el trabajador se convierte en un recurso más valioso dentro de la empresa y aumenta su empleabilidad en el mercado laboral. Al ser personal más empleable en el mercado, la retención del talento se convierte en un desafío para las empresas. En este sentido, el análisis en profundidad de cómo retener el talento puede ser la base de futuras líneas de investigación.

Finalmente, en cuanto a las limitaciones del trabajo, cabe destacar las específicamente vinculadas con la propia metodología de investigación empleada. El estudio de casos, al ser de carácter cualitativo, no permite hacer generalizaciones estadísticas. Por ello, con el objeto de extender y generalizar los resultados, se plantea como futura línea de investigación la realización de estudios cuantitativos con una amplia muestra de empresas que hayan implantado la GT. 
Igualmente, como este estudio se ha realizado en empresas de diferentes tamaños que operan en España, consideramos que sería importante analizar factores de contexto que puedan influir en la forma de orientar e implantar la GT, tales como la industria, el tamaño, el país o la cultura. Estos factores de contexto y los retos asociados a los que se enfrenta una empresa cuando decide poner en marcha esta política, constituyen otra futura línea de investigación. Por último, puede abrirse una tercera línea de investigación adicional orientada a la relación entre la estrategia empresarial, los resultados y la GT. Este proceso puede ser considerando como un antecedente de la estrategia (según la Vía III) y como un moderador de la relación entre la estrategia y los resultados alcanzados.

\section{Referencias}

$\mathrm{Al}$ Ariss, A., Cascio, W. F., \& Paauwe, J. (2014). Talent Management: Current theories and future research direction. Journal of World Business, 49(2), 173-179. https://doi.org/10.1016/j.jwb.2013.11.001

Alonso, A., \& García Muiña, F. E. (2014) La gestión del talento: líneas de trabajo y procesos clave. Intangible Capital, 10(5), 1003-1025. https://doi.org/10.3926/ic.518

Ashton, C., \& Morton, L. (2005). Managing talent for competitive advantage. Strategic HR Review, .(5), 28-31. htt ps://doi.org/10.1108/14754390580000819

Axelrod, B., Handfield-Jones, H., \& Michaels, E. (2002). A new game plan for C players. Harvard Business Review, 80(1), 80-88. https://hbr.org/2002/01/a-new-game-plan-for-c-players

Bardin, L. (1991). Análisis de contenido, vol. 89. Madrid: Ediciones Akal.

Barney, J. (1991). Firm resources and sustained competitive advantage. Journal of Management, 17(1), 99-120. https ://doi.org/10.1177/014920639101700108

Becker, B. E., \& Huselid, M. A. (2006). Strategic human resources management: Where do we go from here? Journal of Management, 32(6), 898-925. https://doi.org/10.1177/0149206306293668

Björkman, I., \& Smale, A. (2010). La gestión global del talento: retos y soluciones. Universia Business Review, Tercer trimestre, 30-42. https://www.redalyc.org/pdf/433/43315583002.pdf

Bonache, J. (1999). El estudio de casos como estrategia de construcción teórica: características, críticas y defensas. Cuadernos de Economía y Dirección de la Empresa, 3, enero-junio, 123-140. https://dialnet.unirioja.es/servlet/ articulo?codigo $=195459$

Boudreau, J. W., \& Ramstad, P. M. (2005a). Talentship, talent segmentation, and sustainability: A new HR decision science paradigm for a new strategy definition. Human Resource Management, 44(2), 129-136. https://doi.org /10.1002/hrm.20054.

Boudreau, J. W., \& Ramstad, P. M. (2005b). Where's your pivotal talent? Harvard Business Review, 83(4), 23-24. ht tps://doi.org/10.1002/hrm.20054

Capelli, P. (2008). Talent on demand: Managing talent in an age of uncertainty. Boston: Harvard Business Press. http s://doi.org/10.1108/ict.2008.03740gae.002

Chuai, X., Preece, D., \& Iles, P. (2008). Is talent management just "old wine in new bottles"? The case of multinational companies in Beijing. Management Research News, 31(12), 901-911. https://doi.org/10.1108/014091708109 20611

Cohn, J. M., Khurana, R., \& Reeves, L. (2005). Growing talent as if your business depended on it. Harvard Business Review, 83(10), 63-70.

Collings, D. G., \& Mellahi, K. (2009). Strategic talent management: A review and research agenda. Human Resource Management Review, 19, 304-313. https://doi.org/10.1016/j.hrmr.2009.04.001

Collings, D. G., Scullion, H., \& Dowling, P. J. (2009). Global staffing: A review and thematic research agenda. International Journal of Human Resource Management, 20, 1251-1269. https://doi.org/10.1080/0958519090 2909806 
Collings, D., Scullion, H., \& Vaiman, V. (2015) Talent management: Progress and prospects. Human Resource Management Review, 25(3), 233-235.

Conger, J. A., \& Fulmer, R. M. (2003). Developing your leadership pipeline. Harvard Business Review, 81(12), 76-84. https://hbr.org/2003/12/developing-your-leadership-pipeline

Dries, N. (2013). The psychology of talent management: A review and research agenda. Human Resource Management Review, 23(4), 272-285. https://doi.org/10.1016/j.hrmr.2013.05.001

Easterby-Smith, M., Thorpe, R., \& Jackson, P. R. (2012). Management research. London: Sage Publications.

Eisenhardt, K. M. (1989). Building Theories from Case Study Research. Academy of Management Review, 14(4), 532-550. https://doi.org/10.5465/amr.1989.4308385

Festing, M., \& Schäfer, L. (2014). Generational challenges to talent management: A framework for talent retention based on the psychological contract perspective. Journal of World Business, 49(2), 262-271. https://doi.org/10 $.1016 / j . j w b .2013 .11 .010$

Frank, F. D., Finnegan, R. P., \& Taylor, C.R. (2004). The race for talent: retaining and engaging workers in the 21st century. People and Strategy, 27(3), 12. http://citeseerx.ist.psu.edu/viewdoc/download?doi=10.1.1.514.4132\& rep=rep1\&type $=$ pdf

Gallardo-Gallardo, E., Nijs, S., Dries, N., \& Gallo, P. (2015). Towards an understanding of talent management as a phenomenon-driven field using bibliometric and content analysis. Human Resource Management Review, 25, 264-279. https://doi.org/10.1016/j.hrmr.2015.04.003

Gandz, J. (2006). Talent development: The architecture of a talent pipeline that works. Ivey Business Journal, 1, 1-4. h ttps://doi.org/10.1108/03090591211192601

Gannon, J.M., \& Maher, A. (2012). Developing tomorrow's talent: the case of an undergraduate mentoring programme. Education + Training, 54(6), 440-455. https://doi.org/10.1108/00400911211254244

Garavan, T. N., Carbery, R., \& Rock, A. (2012). Mapping talent development: Definition, scope and architecture. European Journal of Training and Development, 36(1), 5-24 https://doi.org/10.1108/03090591211192601

Garger, E. M. (1999). Holding on to high performers: A strategic approach to retention. Compensation and Benefits Management, 15(4), 10-17.

Grant, R. M. (1991). The Resource Based Theory of Competitive Advantage: Implications for strategy formulation California Management Review, 33(3), 114-135 https://doi.org/10.2307/41166664

Gunter, K., Bjorkman, I, Farndale, E., Shad, S., Jaap, P., Stiles, P., Trevor, J. \& Wright, P. (2012). Six principles of effective global talent management. MIT Sloan Management Review, 53(2), 25-32. https://www.researchgate.net/profi le/Guenter_Stahl3/publication/254777219.pdf

Hanif, M. I., \& Yunfei, S. (2013). The role of talent management and HR generic strategies for talent retention. African Journal of Business Management, 7(29), 2827-2835. https://doi.org/10.5897/AJBM2012.1369

Hartley, J. F. (1994). Case studies in organizational research. In C. Cassell \& S. Gillian (eds.) Qualitative Methods in Organizational Research. A practical guide (pp. 208-229). London: Sage Publications.

Huselid, M. A., \& Becker, B. E. (1999). An interview with Mike Losey, Tony Rucci, and Dave Ulrich: Three experts respond to HRMJ's special issue on HR strategy in five leading firms. Hum. Resour. Manage, 38: 353-365. http s://doi.org/10.1002/SICI1099-050X19992438:4353::AID-HRM93.0.CO:2-C

Huselid, M. A., Beatty, R. W., \& Becker, B. E. (2005). 'A players' or 'A positions'? The strategic logic of workforce management. Harvard Business Review, December, 110-117. https://hbr.org/2005/12/a-players-or-a-positions -the-strategic-logic-of-workforce-management

Jackson, S. E., \& Schuler, R. S. (1990). Human resource planning: Challenges for industrial/organizational psychologists. American Psychologist, 45(2), 223-239. https://doi.org/10.1037/0003-066X.45.2.223

Joo, B., Sushko, J., \& Mclean, G. (2012). Multiple faces of coaching: Manager as coach, Executive coaching and formal mentoring. Organization Development Journal, 30(1), 19-38. https://pdfs.semanticscholar.org/bc04/c4cad8e8 72d99a8e1c51f0a4994ceed165b0.pdf?_ga=2.67890003.33256447.1586900139-978432319.1586900139 
Kehinde, J., S. (2012). Talent Management: Effect on Organizational Performance. Journal of Management Research, April 1. https://doi.org/10.5296/jmr.v4i2.937

Knox, S., \& Freeman, C. (2006). Measuring and managing employer brand image in the service industry. Journal of Marketing Management, 22(7-8), 695-716. https://doi.org/10.1362/026725706778612103

Kong, E., Chadee, D., \& Raman, R. (2013). Managing Indian IT professionals for global competitiveness: the role of human resource practices in developing knowledge and learning capabilities for innovation. Knowledge Management Research \& Practice, 11(4), 334-345. https://doi.org/10.1057/kmrp.2012.21

Kristof, A. L. (1996). Person-organizational !t: An integrative review of its conceptualisations, measurements and implications. Personnel Psychology, 49, 1-49. https://doi.org/10.1111/j.1744-6570.1996.tb01790.x

Kucherov, D., \& Zavyalova, E. (2012). HRD practices and talent management in the companies with the employer brand. European Journal of Training and Development, 36(1), 86-104. https://doi.org/10.1108/03090591211 192647

Lehman, M.S., Hudson, J. R. Jr., Appley, G. W., Sheehan, E. J. Jr., \& Slevin, D.P. (2011). Modified assessment center approach facilitates organizational change. Journal of Management Development, 30(9), 893-913. https://doi.o $\mathrm{rg} / 10.1108 / 02621711111164367$

Lewis, R. E., \& Heckman, R. J. (2006). Talent management: A critical review. Human Resource Management Review, 16(2), 139-154. https://doi.org/10.1016/j.hrmr.2006.03.001

Martin, G., Gollan, P. J., \& Grigg, K. (2011). Is there a bigger and better future for employer branding? Facing up to innovation, corporate reputations and wicked problems in SHRM. International Journal of Human Resource Management, 22(17), 3618-3637. https://doi.org/10.1080/09585192.2011.560880

Meyers, M. C., van Woerkom, M., \& Dries, N. (2013). Talent - Innate or acquired? Theoretical considerations and their implications for talent management. Human Resource Management Review, 23(4), 305-321. https://doi.o rg/10.1016/j.hrmr.2013.05.003

Michaels, E., Handfield-Jones, H., \& Axelrod, B. (2001). The war for talent. Boston: Harvard Business School Press.

Nijs, S., Gallardo-Gallardo, E., Dries, \& Sels, L., (2014). A multidisciplinary review into the definition, operationalization, and measurement of talent. Journal of World Business, 49, 180-191. https://doi.org/10.101 6/j.jwb.2013.11.002

Pascal, C. (2004). Foreword. In A. Schweyer (Ed.), Talent management systems: Best practices in technology solutions for recruitment, retention, and workforce planning. Canada: Wiley Editions.

Rothwell, W. J. (1994). Effective succession planning: Ensuring leadership continuity and building talent from within. New York: Amacom.

Rynes, S.L., Gerhart, B., \& Parks, L. (2005). Personnel psychology: Performance evaluation and pay for performance. Annual. Review. Psychology, 56, 571-600. https://doi.org/10.1146/annurev.psych.56.091103.070254

Scullion, H. \& Collings, G.D. 2011, Global Talent Management, $1^{\circ}$ edn, Routledge, New York, NY. https://doi.org $/ 10.4324 / 9780203865682$

Scullion, H., Collings, D. G., \& Caligiuri, P. (2010). Global talent management. Journal of World Business, 45(2), 105-108. https://doi.org/10.1016/j.jwb.2009.09.011

Smart, B. D. (1999). Topgrading: How leading companies win by hiring, coaching, and keeping the best people. Paramus, NJ: Prentice Hall Press.

Sullivan, J. (2005). To build, buy or trade talent: That is the question behind talent management. Publicado el 8 de Septiembre de 2005 en http://www.erexchange.com/Articles/default.asp?CID $=\{B 22548 F 2-A A D 0-4551-83 E$ C-561E0AE959EA\}

Tarique, I., \& Schuler, R. S. (2010). Global talent management: Literature review, integrative framework, and suggestions for further research. Journal of World Business, 45(2), 122-133. https://doi.org/10.1016/j.jwb.200 9.09 .019 
Thunnissen, M., Boselie, P., \& Fruytier, B. (2013). Talent management and the relevance of context: Towards a pluralistic approach. Human Resource Management Review, 23(4), 326-336. https://doi.org/10.1016/j.hrmr.2 013.05.004

Walker, J. W., \& LaRocco, J. M. (2002). Talent pools: The best and the rest. Human Resource Planning, 25, 12-14.

Yin, R. K. (1994). Case Study Research. Design and Methods, Applied Social Research Methods Series, Vol. 5, Second Edition. London: Sage Publications.

Yin, R. K. (1998). The abridged version of case study research. In L. Bickman \& D. Rog (eds.) Handbook of Applied Social Research Methods (pp. 229-259). London: Sage Publications.

\section{ANEXO}

\begin{tabular}{|c|c|c|c|c|}
\hline & DIMENSION & & VARIABLES & MEDIDA \\
\hline 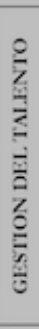 & $\begin{array}{l}\text { VAIOR DE } \\
\text { LAGTPARA } \\
\text { LA EMPRESA }\end{array}$ & 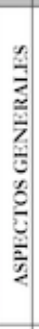 & 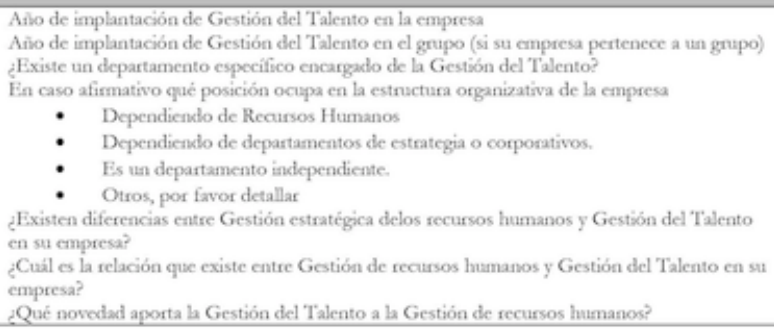 & Nominal \\
\hline \multirow{11}{*}{$\begin{array}{l}\frac{5}{2} \\
\frac{2}{3} \\
\frac{1}{2} \\
\frac{1}{3}\end{array}$} & \multirow{11}{*}{$\begin{array}{l}\text { DIMENSION } \\
\text { PERSONAS } \\
\text { Importancia de } \\
\text { cada fase dentro } \\
\text { del proceso de } \\
\text { GT }\end{array}$} & \multirow[b]{3}{*}{ 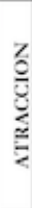 } & Planes de selección especificos para atraes el talento externo & Nominal \\
\hline & & & Planes de selección destinados a la identificación y captación de potenciales de la competencia & Nominal \\
\hline & & & 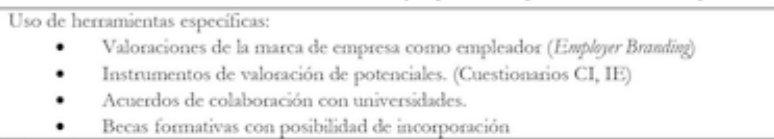 & Nominal \\
\hline & & \multirow[b]{3}{*}{ 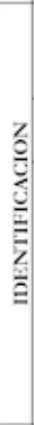 } & $\begin{array}{l}\text { Herramientas de identificación: } \\
\text { Evaluación del desemperio } \\
\text { Evaluación del potencial } \\
\text { Assessment Centro }\end{array}$ & Nominal \\
\hline & & & $\begin{array}{l}\text { Resultados en los programas de información inteno o el criterio de los formadores en la } \\
\text { identificación del talento }\end{array}$ & Nominal \\
\hline & & & 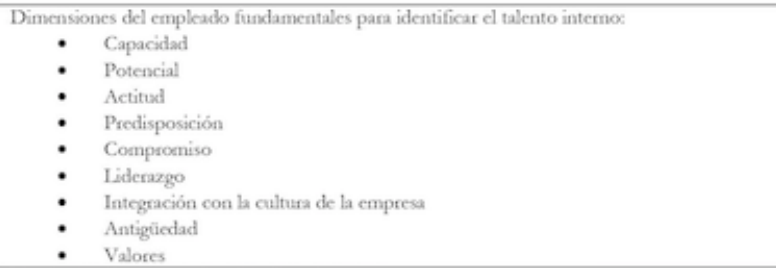 & Nominal \\
\hline & & \multirow[b]{2}{*}{ 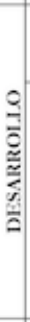 } & $\begin{array}{l}\text { Grado en que el desarrollo del talento busex } \\
\text { - Reforzar las dimensiones del talento mis positivas del individuo. } \\
\text { - Desarrollar las dimensiones del talento que son más débiles en el individuo }\end{array}$ & Nominal \\
\hline & & & 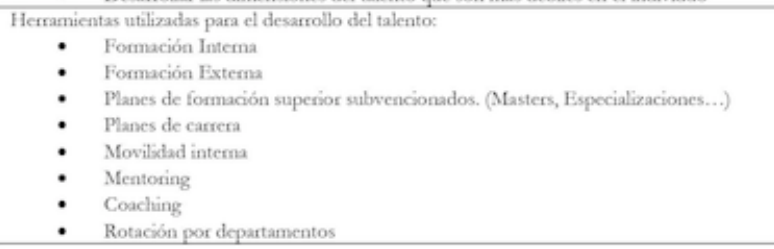 & $\begin{array}{l}\text { Ordinal } \\
\text { Escala } \\
\text { Likest 1-5 }\end{array}$ \\
\hline & & \multirow{3}{*}{ 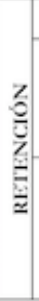 } & ¿Posee la empresa una media sahrial suyerior al mescado? & Nominal \\
\hline & & & $\begin{array}{l}\text { PRÁCTICAS DIFERENCIADORAS } \\
\text { Desplicgue del talento acorde a sus peticiones } \\
\text { Politicas retributivas superiores a la media de la posición } \\
\text { Politicas de flexibilidad y y conciliación superiores a la media de la posición } \\
\text { Motivación de potenciales }\end{array}$ & Nominal \\
\hline & & & $\begin{array}{l}\text { CAPACIDAD DE RETENCIÓN DE: } \\
\text { La retribución económica } \\
\text { Las politicas de conciliación } \\
\text { La existencia de planes de carrera y promoción interna } \\
\text { La motivación } \\
\text { El entorno de trabajo }\end{array}$ & $\begin{array}{c}\text { Ordinal } \\
\text { Escala } \\
\text { Likest 1-5 }\end{array}$ \\
\hline
\end{tabular}




\begin{tabular}{|c|c|c|c|}
\hline \multirow[t]{4}{*}{$\begin{array}{l}\text { DIMENSION } \\
\text { PUESTOS } \\
\text { CLAVE }\end{array}$} & \multirow{4}{*}{ 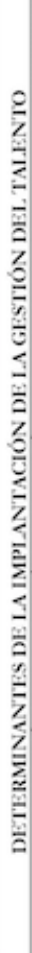 } & 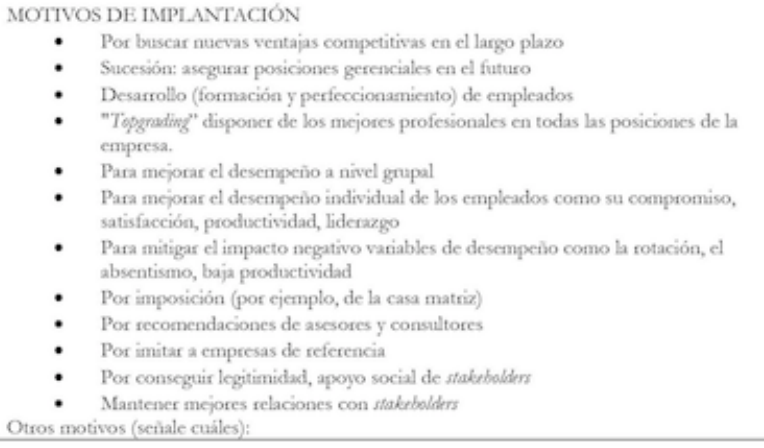 & $\begin{array}{c}\text { Ordinal } \\
\text { Escala } \\
\text { Likert1-5 }\end{array}$ \\
\hline & & $\begin{array}{l}\text { OBJETTVOS DE IMPLANTACIÓN } \\
\text { Individuales o grupales u organizativos } \\
\text { Económicos o competitivos o sociales } \\
\text { Estratígicos (medio y largo plazo) o tícticos (conto plazo) } \\
\text { Impulsar la innovación }\end{array}$ & Nominal \\
\hline & & $\begin{array}{l}\text { CONDICIONANIES DE IMPLANTACIÓN } \\
\text { Compromiso alta dirección } \\
\text { Estrategia de la empresa (plan estratégico o cambio estratćgico) } \\
\text { Estruetura } \\
\text { Cultura } \\
\text { Confidencialidad }\end{array}$ & Nominal \\
\hline & & $\begin{array}{l}\text { OBSTÁCULOS DE IMPLANTACIÓN } \\
\text { Las relaciones laborales. } \\
\text { La importancia de la equidad interna. } \\
\text { La complejidad derivada de coordinar a los empleados de áreas de distinto signo. } \\
\text { Resistencia interma al cambio de los diferentes lideres de la empresa. } \\
\text { Resistencia interna al cambio en los empleados base de la empresa. } \\
\text { La dificultad por parte de algunos empleados de lidear con mayores grados de incertidumbre. } \\
\text { El fracaso por parte de algunos empleados a la hora de liderar equipos. } \\
\text { Otros }\end{array}$ & $\begin{array}{c}\text { Ordinal } \\
\text { Escala } \\
\text { L.jkert1-5 }\end{array}$ \\
\hline
\end{tabular}

\section{Notas}

* Artículo de investigación científica.

\section{Licencia Creative Commons CC BY 4.0}

Sugerencia de citación: de la Calle-Durán, M.C, García M., F. E. y Osuna, A. A. (2020). Análisis de la gestión del talento en función de sus dimensiones y procesos. Un estudio de casos. Cuadernos de Administración, 33. https://doi.org/10.11144/Javeriana.cao33.agtfdp 